\title{
13. THE BALLENA AND DELFIN WELLS OFF CENTRAL PERU: REVISED AGES ${ }^{1}$
}

\author{
Hans Schrader ${ }^{2}$ and José Cruzado Castaneda ${ }^{3}$
}

\begin{abstract}
Diatomaceous samples from the middle portions of two industrial wells (Ballena and Delfin) drilled by Occidental Petroleum Co. offshore central Peru yielded biostratigraphic interpretations that made substantial revision of the biostratigraphic ages of these portions necessary. Most of the sequences formerly dated Eocene-Oligocene now are of middle Miocene to late Miocene age.
\end{abstract}

\section{INTRODUCTION}

The Delfin and Ballena wells were drilled on the continental shelf and upper continental slopes near $9^{\circ} \mathrm{S}$ in 1974 (Figs. 1 and 2). The Ballena well is located above the crest of the outer shelf high, and the Delfin well is located on the eastern flank of the Trujillo Basin. Both wells penetrated a sequence of marine Tertiary sediments and reached Paleozoic or Precambrian metamorphic basement (Fig. 2).

The Delfin well penetrated $2667 \mathrm{~m}$ and was drilled in at least $500 \mathrm{~m}$ of Eocene strata underlying Miocene marine mudstones. The Eocene section can be correlated to the Chira Formation of northwestern Peru.

Previous correlation of the stratigraphic sequence in both wells could not be tied to similar sequences in northwestern Peru or to the Pisco Basin stratigraphy. These industrial wells drilled by Occidental Petroleum Co. were used in the past by several scientists to correlate stratigraphic reflectors and basement ages to the two wells drilled off the coast of Peru (Kulm et al., 1981, 1982; Hussong et al., 1985). These scientists assumed that the middle portions of the wells are of Oligocene/Eocene age.

\section{METHODS}

We had access to several samples from the central portion of both wells (courtesy of Petróleos del Peru). All samples and their diatom species compositions for the Ballena well are listed in Table 1. In addition, we list in Table 2 samples from the Delfin well that were barren of siliceous microfossils.

Slides were prepared using the techniques of Schrader and Gersonde (1978). These were mounted in Hyrax and scanned through at high power using oil immersion planapochromatic Zeiss objectives.

The diatom biostratigraphy proposed by Barron (1985) from the equatorial Pacific, which is directly calibrated to the magnetic reversal stratigraphy, was used exclusively. Most major Pacific diatom zones, as defined by Barron (1985), could be recognized in sites drilled off the Peruvian margin during Leg 112 (see this volume and Suess, von Huene, et al., 1988).

An earlier draft of this manuscript was made available during the Leg 112 drilling campaign off Peru in 1986 and was extensively used by the Shipboard Scientific Party and shore-based investigators, tying the Ballena and Delfin record to the Leg 112 results (see Suess, von Huene, et al., 1988).

\footnotetext{
${ }^{1}$ Suess, E., von Huene, R., et al., 1990. Proc. ODP, Sci. Results, 112: College Station, TX (Ocean Drilling Program).

2 Geological Institute, Avd. B, University of Bergen, Allegaden 41, N-5007 Bergen, Norway, Peru.
}

\section{RESULTS}

\section{Analysis of Data}

All species encountered and their abundances are listed in the tables. Biostratigraphically important species are illustrated in Plates 1 and 2.

\section{Ballena Well}

Samples 1 through 4 (Table 1) contained a well-preserved and diversified diatom assemblage typical for the Peruvian margin, with a large amount of neritic and true coastal upwelling indicator species and some admixtures of displaced shallow-water benthic diatoms (see Table 3 for a detailed floral analysis).

\section{Sample 1}

No recognizable reworked species were observed. Triceratium aff. cinnamomeum is a species that occurs also in marine fossil deposits from Moron, Spain, and in tropical Pacific sediments of middle Miocene age (Barron, 1985). Delphineis aff. ossiformis (a Miocene form) is a new species that has a bonelike shape; it differs from its Quaternary counterpart in its fine structure. This species is not formally described here; Coscinodiscus yabei Zone of Barron (1985), late Miocene, about 7.65 to $8.9 \mathrm{Ma}$.

\section{Sample 2}

No reworked species were encountered; Craspedodiscus coscinodiscus Zone of Barron (1985), equivalent to the Nitzschia denticuloides Zone of Weaver and Gombos (1981), middle Miocene, around 10.7 to $11.8 \mathrm{Ma}$.

\section{Sample 3}

Synedra jouseana is probably reworked; Coscinodiscus lewisianus Zone of Barron (1985) to Denticulopsis hustedtii/Denticulopsis lauta Zone, Subzone a of Barron (1980) and Corbisema triacantha Zone of Locker and Martini (1986), Distephanus stauracanthus horizon, middle Miocene, about 12.9 to $14.2 \mathrm{Ma}$.

\section{Sample 4}

Nitzschia aff. porteri, with a few displaced shallow-water benthic species. Based on the occurrence of Denticulopsis nicobarica s.l., Distephanus stauracanthus, Synedra miocenica, and Rhizosolenia miocenica, this sample is tentatively assigned to the Denticulopsis nicobarica Zone of Barron (1985), early Miocene, 17.8 to $16.4 \mathrm{Ma}$. Thalassiosira fraga, which approximates the base of the following Cestodiscus peplum Zone, was not observed. The middle Miocene $\mathrm{Di}$ stephanus stauracanthus horizon (Locker and Martini, 1986) is younger than this occurrence. 


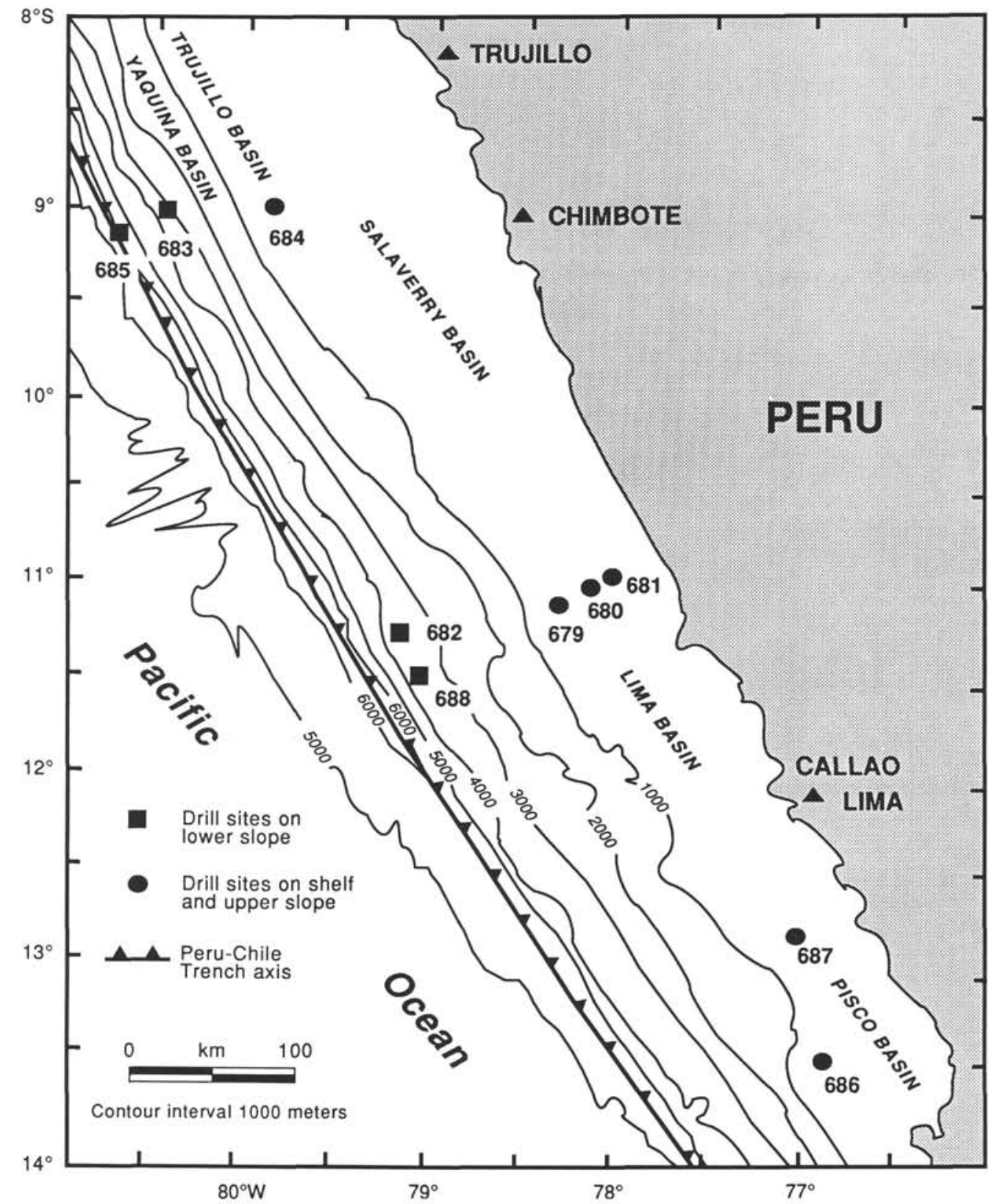

Figure 1. Location map of the Ballena and Delfin wells and ODP Leg 112 drilling sites off the coast of Central Peru (from Suess, von Huene, et al., 1988). Major forearc basins based on Thornburg and Kulm (1981).

Sedimentation rates are about $30 \mathrm{~m} / \mathrm{m} . \mathrm{y}$.

The occurrence of species that are abundantly found in the southernmost part of the Pacific Ocean, e.g., during DSDP Leg 35 (Schrader, 1976; Ciesielski, 1983) is unique during this middle Miocene period. The precursor of the Humboldt Current must have been intensified to enable transport of truly Antarctic floral elements that far north. Strong upwelling phenomena are evident by the extremely well-preserved diatom assemblages and species composition with Thalassionema nitzschioides, Delphineis species, Chaetoceros resting spores, and Skeletonema species, among many others.

\section{Delphin Well}

Twenty-three samples were analyzed; of these only three samples contained traceable amounts of marine diatoms, whereas all other samples were barren in siliceous microfossils.

The three samples that contained diatoms were Sample 5 at $2740-2750 \mathrm{ft}, 837.2 \mathrm{mbsf}$; Sample 6 at $2640-2650 \mathrm{ft}, 806.7 \mathrm{mbsf}$; and Sample 7 at $2130-2140 \mathrm{ft}, 651.2$ mbsf.

Denticulopsis nicobarica s.l. disappears from the equatorial Pacific around $12.5 \mathrm{Ma}$; the first occurrence of Rhizosolenia miocenica in the Pacific is around $13.5 \mathrm{Ma}$; Denticulopsis hustedtii became established in the tropical Pacific around $13.9 \mathrm{Ma}$ (Barron, 1985). Based on the presence of Denticulopsis hustedtii, Denticulopsis nicobarica s.l., Rhizosolenia miocenica, and Nitzschia denticuloides, these samples can be placed into the Nitzschia denticuloides to the lowermost Nitzschia grossepunctata Zones of Weaver and Gombos (1981), which correlates in part to the Coscinodiscus lewisianus through Craspedodiscus 


\section{INDUSTRY WELLS}
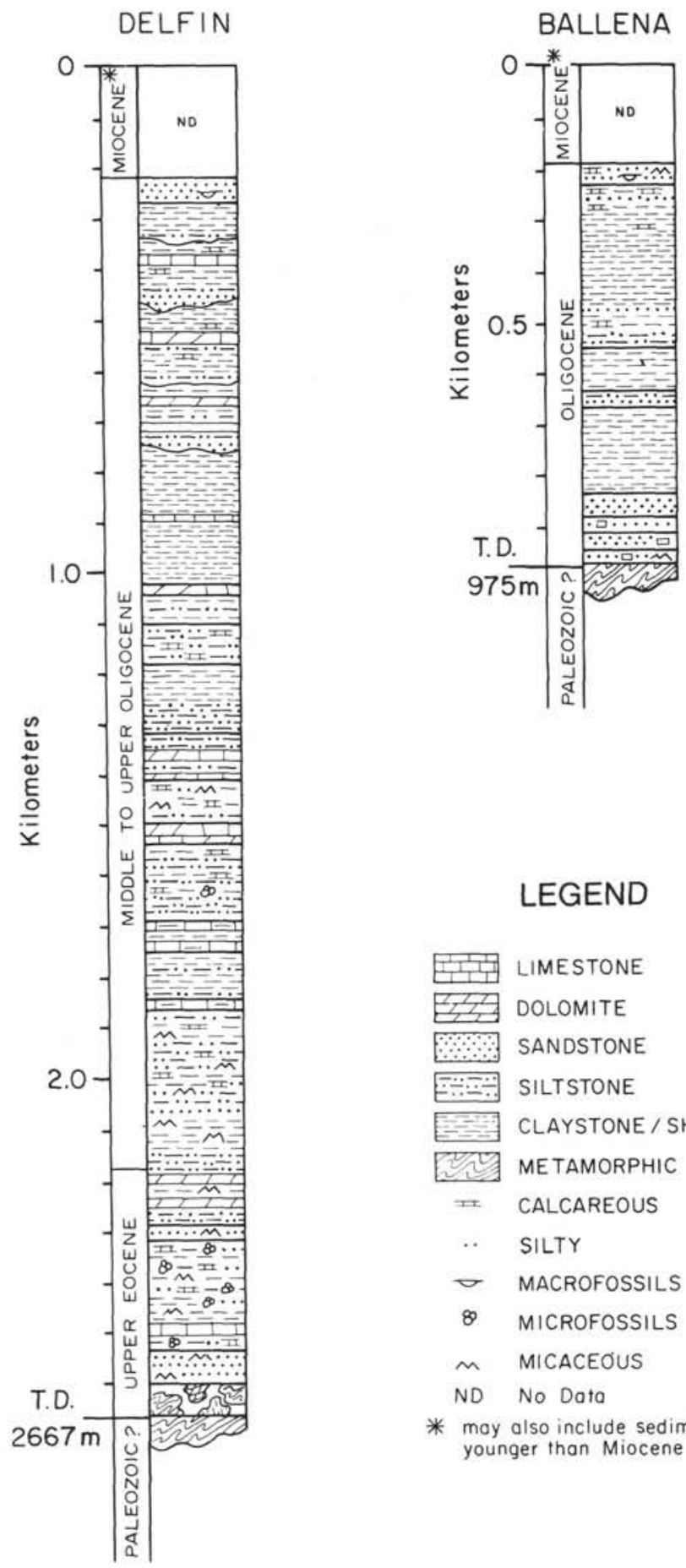

Figure 2. Industry drill holes of the central Peruvian forearc near $9^{\circ} \mathrm{S}$ latitude; stratigraphic columns and sedimentology are from Kulm et al. (1985) with biostratigraphic ages as originally published. Figure taken from Kulm et al., 1988.

coscinodiscus Zones of Barron (1985) and is of middle Miocene age, 14.2 to $10.7 \mathrm{Ma}$. Further refinement of the biostratigraphic interpretation is impossible because of the poor preservation and scarcity of diatom floras.

Based on biostratigraphic ages and floral composition, a correlation between the two wells may be possible for the 300
Table 1. Diatomaceous samples from the Ballena well, off Peru.

\begin{tabular}{ccc}
\hline Sample & $\begin{array}{c}\text { Depth } \\
\text { (ft) }\end{array}$ & $\begin{array}{c}\text { Depth } \\
\text { (mbsf) }\end{array}$ \\
\hline 1 & $890-900$ & $271.45-274.50$ \\
2 & $1180-1190$ & $359.90-362.95$ \\
3 & $1280-1290$ & $390.40-393.45$ \\
4 & $1630-1640$ & $497.15-500.20$ \\
\hline
\end{tabular}

Table 2. Samples barren in diatoms from the Delfin well.

\begin{tabular}{cr}
\hline $\begin{array}{c}\text { Depth } \\
\text { (ft) }\end{array}$ & $\begin{array}{c}\text { Depth } \\
\text { (mbsf) }\end{array}$ \\
\hline $5590-5600$ & 1706.5 \\
$5580-5590$ & 1703.4 \\
$5560-5570$ & 1697.3 \\
$5550-5560$ & 1694.3 \\
$5530-5540$ & 1688.2 \\
$5520-5530$ & 1685.1 \\
$5510-5520$ & 1682.1 \\
$5500-5510$ & 1679.0 \\
$5100-5110$ & 1557.0 \\
$5090-5100$ & 1554.0 \\
$5070-5080$ & 1547.9 \\
$5060-5070$ & 1544.8 \\
$5550-5560$ & 1694.3 \\
$4900-4910$ & 1496.0 \\
$2770-2780$ & 846.4 \\
$2760-2770$ & 843.3 \\
$2750-2760$ & 840.3 \\
$2650-2660$ & 808.3 \\
$2630-2640$ & 803.7 \\
$2620-2630$ & 800.6 \\
\hline &
\end{tabular}

to 450 mbsf in the Ballena well to the 600 to $800 \mathrm{mbsf}$ in the Delfin well (Fig. 3).

\section{ACKNOWLEDGMENTS}

We thank PetroPeru for providing the samples and allowing us to publish this paper. The Norwegian Council for Humanities and Research funded this project.

\section{REFERENCES}

Barron, J. A., 1980. Miocene to Quaternary diatom biostratigraphy of DSDP Leg 57, off northeast Japan. In Shipboard Scientific Party, Init. Repts. DSDP, 56, 57 (2): Washington (U. S. Govt. Printing Office), 641-685.

1985. Late Eocene to Holocene diatom biostratigraphy of the equatorial Pacific Ocean, Deep Sea Drilling Project Leg 85. In Meyer, L., Theyer, F., Thomas, E., et al., Init. Repts. DSDP, 85: Washington (U.S. Govt. Printing Office), 413-456.

Ciesielski, P. F., 1983. The Neogene and Quaternary diatom biostratigraphy of subantarctic sediments, Deep Sea Drilling Project 71. In Ludwig, W. J., Krasheninnikov, V. A., et al., Init. Repts. DSDP, 71: Washington (U.S. Govt. Printing Office), 635-665.

Hussong, D. M., Dang, S. P., Kulm, L. D., Couch, R. W., and Hilde, T.W.C. (eds.) 1985. Atlas of the Ocean Margin Drilling Program, Regional Atlas Series 9, Peru Chile Trench off Peru: Woods Hole (Marine Sci. Int.).

Kulm, L. D., Dymond, J., Dasch, E. J., and Hussong, D. M. (Eds.), 1981a. Nazca Plate: Crustal Formation and Andean Convergence: Boulder (Geol. Soc. Am. Mem.), 154.

Kulm, L. D., Schrader, H., Resig, J., Thornburg, T., Masias, A., and Johnson, L., 1981b. Late Cenozoic carbonates on the Peru continental margin: Lithostratigraphy, biostratigraphy, and tectonic history. Geol. Soc. Am. Mem., 154:469-508. 
Kulm, L. D., Resig, J., Thornburg, T., and Schrader, H., 1982. Cenozoic structure, stratigraphy and tectonics of a fast convergence zone: Central Peru forearc. Symp.: Trench and Forearc Sedimentation and Tectonics in Modern and Ancient Subduction Zones (London), 151-169.

Kulm, L. D., Thornburg, T., and Dang, S., 1985. Drill hole stratigraphy, dredge lithologies, and sample locations. In Hussong, D. M., et al. (Eds.), Atlas of the Ocean Margin Drilling Program, Regional Atlas Series 9, Peru-Chile Trench off Peru: Woods Hole (Mar. Sc. Int.).

Kulm, L. D., Thornburg, T. M., Suess, E., Resig, J., and Fryer, P., 1988. Clastic, diagenetic, and metamorphic lithologies of a subsiding continental block: Central Peru Forearc. In Suess, E., von Huene, R., et al., Proc. ODP, Init. Repts., 112: College Station, TX (Ocean Drilling Program), 91-107.

Locker, S., and Martini, E., 1986. Silicoflagellates and some sponge spicules from the southwest Pacific, Deep Sea Drilling Project, Leg 90. In Kennett, J. P., von der Borch, C. C., et al., Init. Repts. DSDP, 90: Washington (U.S. Govt. Printing Office), 887-924.
Schrader, H., 1976. Cenozoic planktonic diatom biostratigraphy of the southern Pacific Ocean. In Hollister, C. D., Craddock, C., et al.,1976, Init. Repts. DSDP, 35: Washington (U.S. Govt. Printing Office), 605-671.

Schrader, H., and Gersonde, R., 1978. Diatoms and silicoflagellates in the eight meter section of the lower Pliocene of the Capo Rossello. Utrecht Micropaleontol. Bull., 17:129-176.

Suess, E., von Huene, R., et al., 1988. Proc. ODP, Init. Repts., 112: College Station, TX (Ocean Drilling Program).

Thornburg, T. M., and Kulm, L. V., 1981. Sedimentary basins of the Peru continental margin: structure, stratigraphy, and Cenozoic tectonics from $6^{\circ} \mathrm{S}$ to $16^{\circ} \mathrm{S}$ latitude. Geol. Soc. Am. Mem., 154:393-422.

Weaver, F. M., and Gombos, A. M., 1981. Southern high-latitude diatom biostratigraphy. Spec. Publ. Soc. Econ. Paleontol. Mineral., 32:445-470.

Date of initial receipt: 5 January 1989

Date of acceptance: 5 July 1989

Ms 112B-187 
Table 3. Diatom and silicoflagellate species found in diatom bearing samples from the two industrial wells "Ballena and Delfin".

\begin{tabular}{|c|c|c|c|c|c|c|c|}
\hline & & & & Sampl & & & \\
\hline Species & 1 & 2 & 3 & 4 & 5 & 6 & 7 \\
\hline Abundance & A & A & A & A & $\mathbf{R}$ & $\mathbf{R}$ & $\mathrm{F}$ \\
\hline Preservation & $\mathrm{E}$ & E & $\mathrm{E}$ & E & $\mathrm{P}$ & M & M \\
\hline Diatoms: & & & & & & & \\
\hline Actinocyclus ellipticus var. & $\mathbf{R}$ & - & - & - & - & - & - \\
\hline Actinocyclus ellipticus & $\mathrm{R}$ & - & - & - & - & - & - \\
\hline Actinocyclus ehrenbergii & $\mathrm{R}$ & - & $\mathbf{R}$ & $\mathrm{R}$ & - & - & - \\
\hline Actinocyclus ingens & - & $\mathrm{R}$ & $\mathbf{R}$ & $\mathrm{R}$ & - & - & $\mathbf{R}$ \\
\hline Annellus californicus & - & - & $\mathrm{R}$ & - & - & - & - \\
\hline Actinoptychus undulatus & $\mathrm{F}$ & $\mathbf{R}$ & $\mathbf{R}$ & $\mathbf{R}$ & $\mathrm{R}$ & $\mathbf{R}$ & $\mathbf{R}$ \\
\hline Actinoptychus splendens & $\mathrm{R}$ & $\mathrm{R}$ & - & $\mathrm{R}$ & - & $\mathbf{R}$ & $\mathbf{R}$ \\
\hline Cestodiscus pulchellus & - & - & $\mathrm{F}$ & $\mathrm{R}$ & - & - & - \\
\hline Azpeitia aff. tabularis & - & $\mathbf{R}$ & - & - & - & - & $\mathbf{R}$ \\
\hline Coscinodiscus deformans & - & $\mathbf{R}$ & - & - & - & - & - \\
\hline Coscinodiscus endoi & - & $\mathbf{R}$ & $\mathbf{R}$ & - & - & - & - \\
\hline Azpeitia nodulifer & $\mathbf{R}$ & - & - & - & - & - & - \\
\hline Coscinodiscus marginatus & - & $\mathrm{R}$ & - & - & - & - & $\mathrm{R}$ \\
\hline Coscinodiscus symbolophorus & $\mathrm{R}$ & $\mathbf{R}$ & $\mathbf{R}$ & $\mathbf{R}$ & - & $\mathbf{R}$ & - \\
\hline Azpeitia vetustissimus & - & $\mathbf{R}$ & $\mathrm{R}$ & - & - & - & - \\
\hline Azpeitia v. var. javanicus & - & - & $\mathrm{R}$ & - & - & - & - \\
\hline Craspedodiscus coscinodiscus & - & $\mathbf{R}$ & - & $\mathrm{R}$ & - & - & - \\
\hline $\begin{array}{l}\text { Delphineis "ossiformis" } \\
\text { (Miocene)R }\end{array}$ & $\mathbf{R}$ & - & - & - & - & - & \\
\hline Delphineis aff. karstenii & $\mathrm{R}$ & - & - & - & - & - & $\mathbf{R}$ \\
\hline Denticulopsis antarctica & - & $\mathbf{R}$ & $\mathbf{R}$ & - & - & - & - \\
\hline Denticulopsis hustedtii & $\mathrm{C}$ & $\mathrm{C}$ & C & $\mathrm{F}$ & $\mathbf{R}$ & $\mathbf{R}$ & - \\
\hline Denticulopsis nicobarica s.I. & - & C & C & $\mathrm{F}$ & $\mathbf{R}$ & $\mathbf{R}$ & - \\
\hline Denticulopsis punctata & - & - & $\mathbf{R}$ & - & - & - & - \\
\hline Hemiaulus aff. polymorphus & - & $\mathbf{R}$ & - & $\mathrm{R}$ & - & - & - \\
\hline Hemidiscus cuneiformis & $\mathrm{R}$ & - & - & - & - & - & - \\
\hline Nitzschia aff. praereinholdii & $\mathrm{R}$ & - & - & - & - & - & - \\
\hline Nitzschia denticuloides & $\mathrm{R}$ & - & - & $\mathrm{R}$ & - & - & $\mathbf{R}$ \\
\hline Nitzschia aff. porteri & - & $\mathbf{R}$ & - & $\mathrm{R}$ & - & - & - \\
\hline Nitzschia spec. 1 & $\mathrm{~F}$ & $\mathrm{R}$ & $\mathrm{R}$ & - & - & - & - \\
\hline Mediaria splendida & $\mathrm{R}$ & $\mathbf{R}$ & $\mathrm{F}$ & - & - & - & - \\
\hline Paralia sulcata & $\mathrm{R}$ & $\mathrm{R}$ & $\mathrm{R}$ & $\mathrm{R}$ & - & $\mathbf{R}$ & - \\
\hline Pseudodimerogramma elegans & $\mathrm{R}$ & - & $\mathrm{R}$ & - & - & $\mathbf{R}$ & - \\
\hline Rhizosolenia alata & $\mathrm{R}$ & - & - & - & - & - & - \\
\hline Rhizosolenia miocenica & - & - & - & $\mathbf{R}$ & - & - & $\mathbf{R}$ \\
\hline Rhizosolenia s & - & $\mathbf{R}$ & $\mathbf{R}$ & $\mathrm{R}$ & - & - & - \\
\hline Rossiella praepaleacea & $\mathbf{R}$ & $\mathbf{R}$ & $\mathbf{R}$ & - & - & - & - \\
\hline Rouxia californica & - & $\mathbf{R}$ & - & $\mathrm{R}$ & - & - & - \\
\hline Rouxia aff. peragallii & - & $\mathbf{R}$ & - & $\mathbf{R}$ & - & - & - \\
\hline diploneides & $\mathrm{R}$ & $\mathbf{R}$ & - & - & - & - & - \\
\hline Skeletonema spec. 1 & $\mathrm{R}$ & $\mathrm{R}$ & $\mathrm{R}$ & $\mathrm{R}$ & - & - & - \\
\hline Stephanopyxis aff. palmeriana & - & $\mathbf{R}$ & - & $\mathrm{R}$ & - & - & - \\
\hline Stephanopyxis aff. turris & $\mathrm{R}$ & $\mathrm{R}$ & $\mathrm{R}$ & $\mathrm{R}$ & - & - & $\mathbf{R}$ \\
\hline Synedra jouseana & - & - & $\mathrm{R}$ & - & - & - & - \\
\hline Synedra miocenica & $\mathrm{R}$ & $\mathbf{R}$ & $\mathrm{R}$ & $\mathbf{R}$ & - & - & - \\
\hline Thalassionema nitzschic & C & C & C & C & C & $\mathrm{C}$ & C \\
\hline Thalassionema nitz. var. parva & C & $\mathrm{F}$ & $\mathrm{F}$ & $\mathrm{F}$ & C & $\mathbf{R}$ & - \\
\hline Thalassiosira leptopus & $\mathrm{R}$ & $\mathbf{R}$ & $\mathbf{R}$ & - & - & - & - \\
\hline Thalassiosira miocenica & $\mathrm{R}$ & - & - & - & - & - & - \\
\hline Thalassiosira plicata & $\mathrm{R}$ & $\mathbf{R}$ & - & - & - & - & - \\
\hline Thalassiosira yabei & $\mathbf{R}$ & $\mathbf{R}$ & - & - & - & - & - \\
\hline Thalassiothrix longissima & $\mathrm{F}$ & $\mathrm{F}$ & $\mathrm{C}$ & C & - & $\mathrm{C}$ & $\mathrm{C}$ \\
\hline Thalassiothrix robusta & - & - & $\mathbf{R}$ & F & - & $\mathbf{R}$ & - \\
\hline Triceratium aff. cinnamomeum & - & $\mathbf{R}$ & - & - & - & - & - \\
\hline & & & & & & & \\
\hline Paramesocena apiculata & $\mathbf{R}$ & - & - & $\mathbf{R}$ & - & - & - \\
\hline Distephanus stauracanthus & - & - & - & F & - & - & - \\
\hline Distephanus crux & $\mathrm{R}$ & $\mathbf{R}$ & $\mathbf{R}$ & $\mathbf{R}$ & $\mathrm{T}$ & $\mathbf{R}$ & $\mathrm{T}$ \\
\hline
\end{tabular}

Abundance of diatoms and silicoflagellates in $\mathrm{R}=$ rare, $\mathrm{F}=$ few, $\mathrm{C}=$ common, $\mathrm{A}=$ abundant; preservation expressed as $\mathrm{P}=$ poor, $\mathrm{M}=$ moderate, and $\mathrm{E}=$ excellent.

\section{INDUSTRY WELLS}

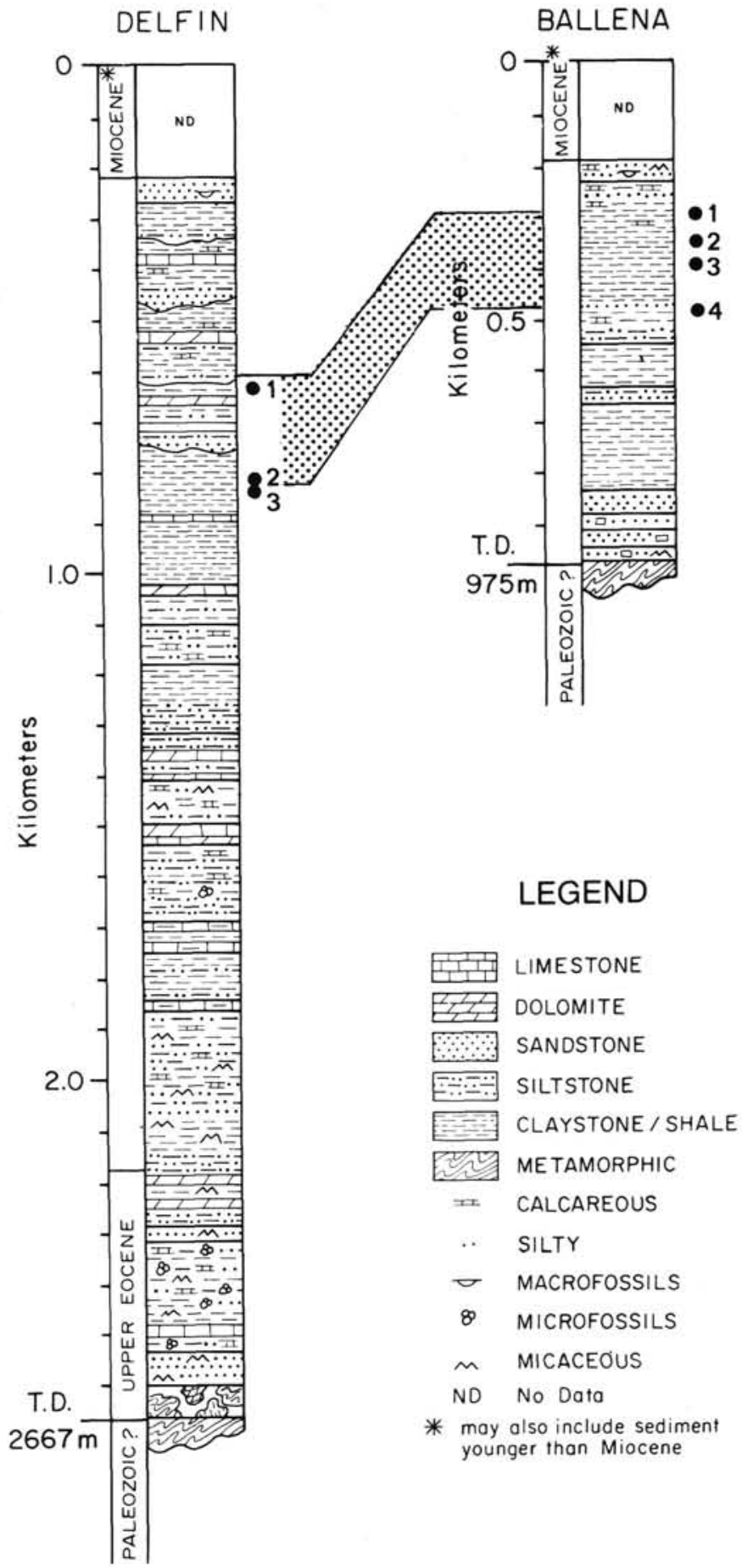

Figure 3. Ballena and Delfin Wells. Location of diatom bearing samples, and proposed correlation of the 600 to $800 \mathrm{~m}$ section of the Delfin Well with the 300 to $450 \mathrm{~m}$ section of the Ballena Well based on diatom zonal assignments. 


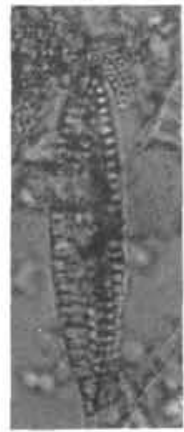

1

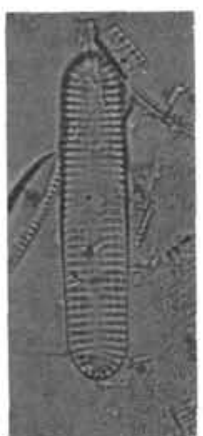

7

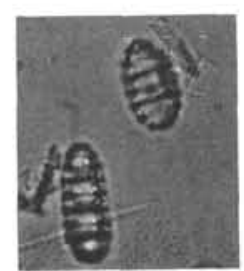

14

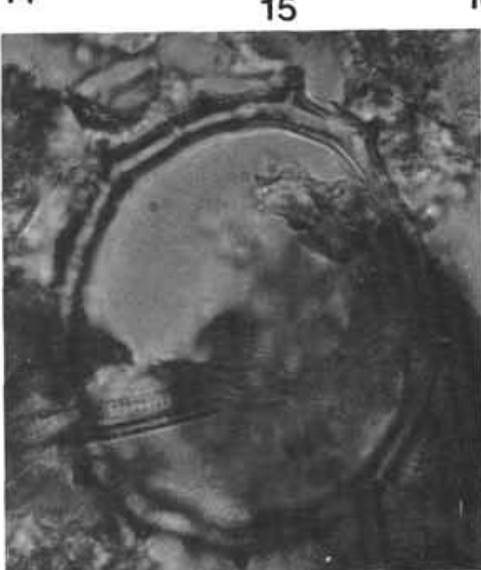

21

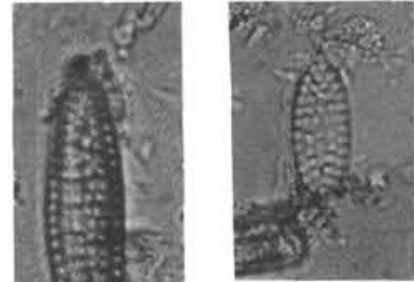

3

\section{2}

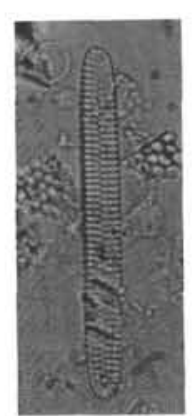

8

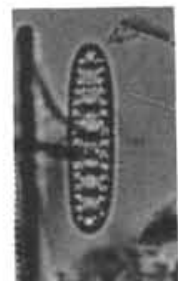

15

6

9
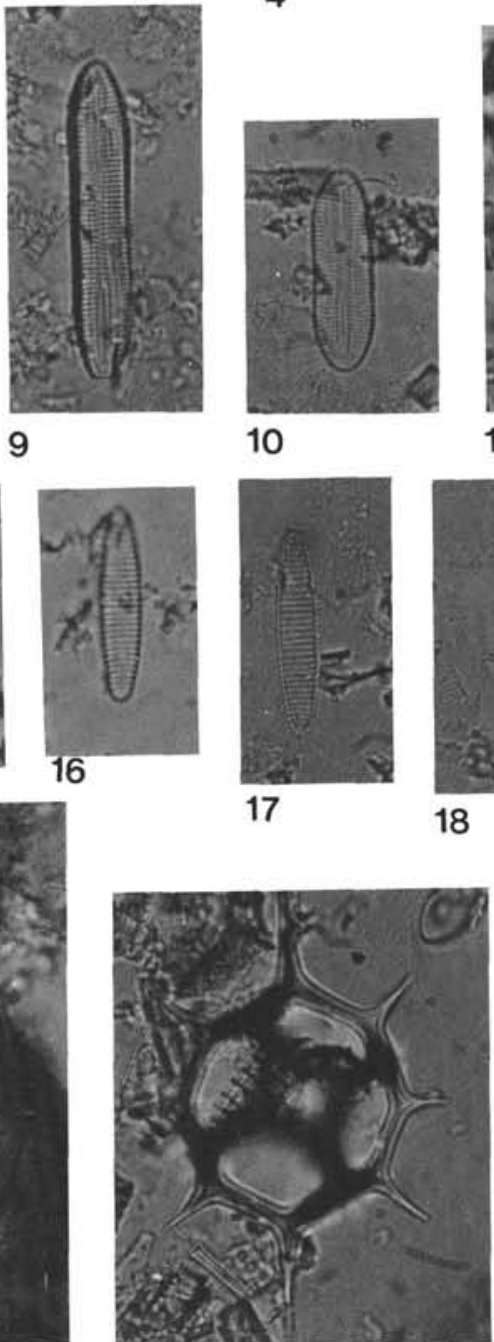

22

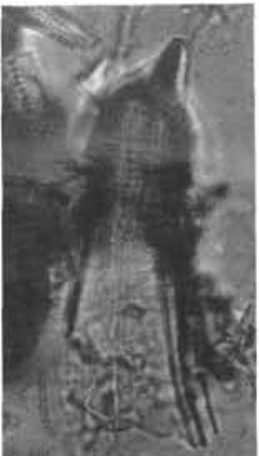

4

17

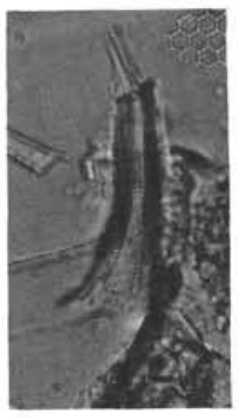

5

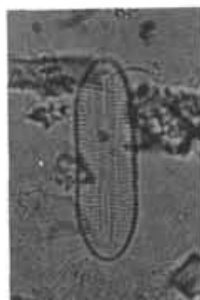

10

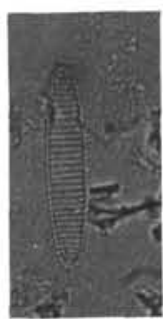

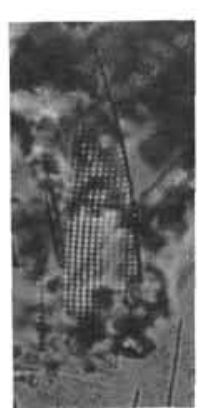

11

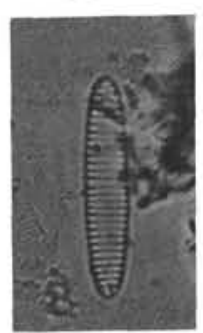

18
19

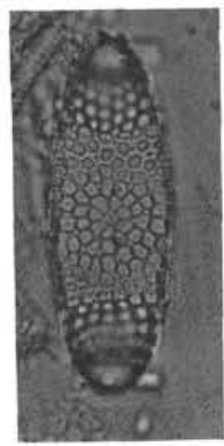

6

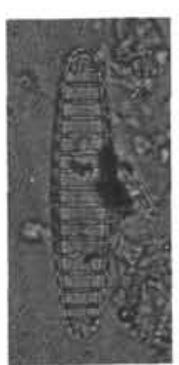

12

13

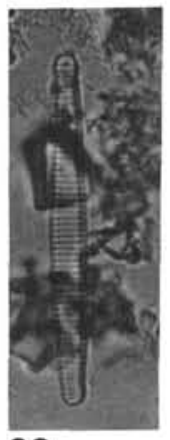

20

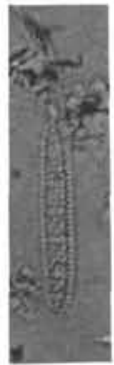

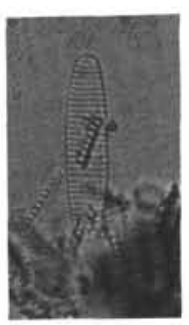

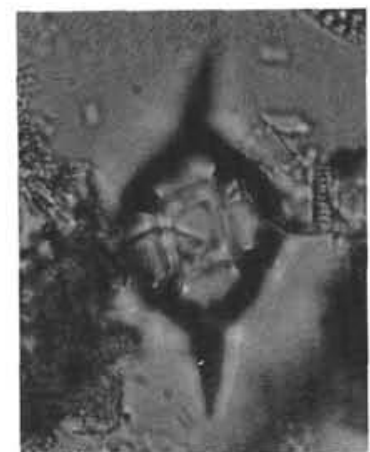

23

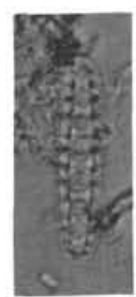

24

Plate 1. 1-3. Rossiella praepaleacea Schrader and Gersonde. 4, 5. Rhizosolenia miocenica Schrader. 6. Hemiaulus aff. polymorphus. 7. Delphineis sp.(new species, not formally described here). 8. Pseudodimerogramma elegans Schrader. 9, 10. Rouxia aff. diploneides Schrader. 11. Mediaria splendida Sheshukova-Poretzkaya. 12. Denticulopsis antarctica (McCollum) Simonsen. 13. Denticulopsis nicobarica s.l. (Grunow) Simonsen. 14, 15. Denticulopsis hustedtii (Simonsen and Kanaya) Simonsen. 16-20. Nitzschia sp. (several new species, not formally described here). 21. Silicoflagellate Paramesocena apiculata (Lemmermann) Locker and Martini. 22. Silicoflagellate Distephanus stauracanthus Ehrenberg forma stauracanthus. 23. Silicoflagellate Distephanus crux Ehrenberg subspecies crux Ehrenberg. 24. Denticulopsis hustedtii (Simonsen and Kanaya) Simnosen. 


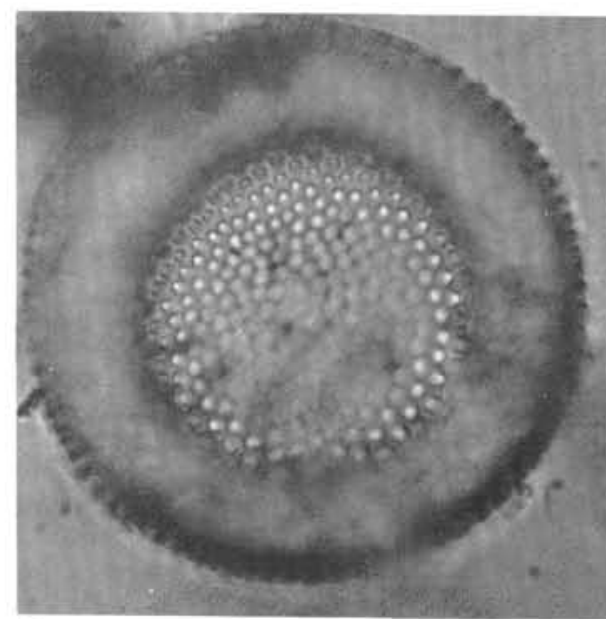

1

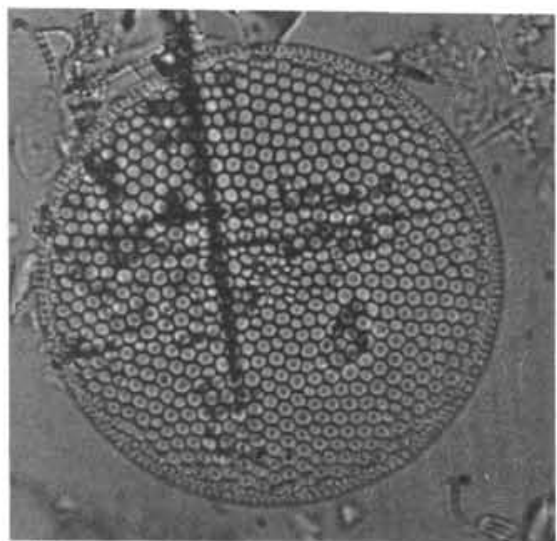

4

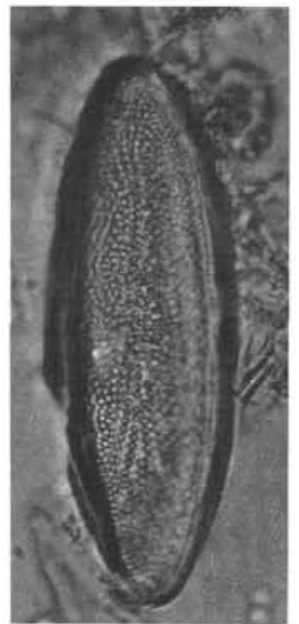

8
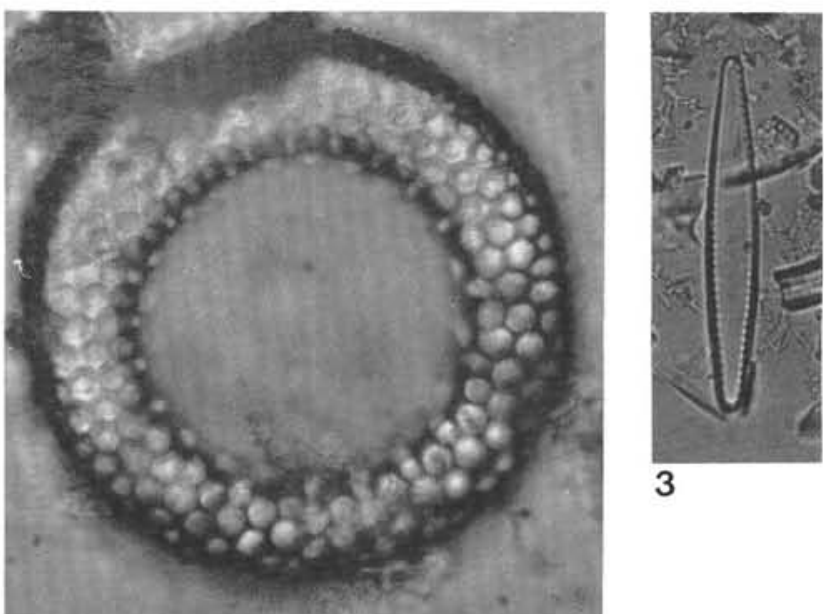

3

2

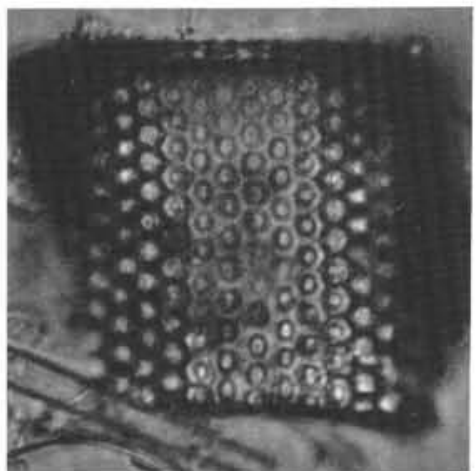

5

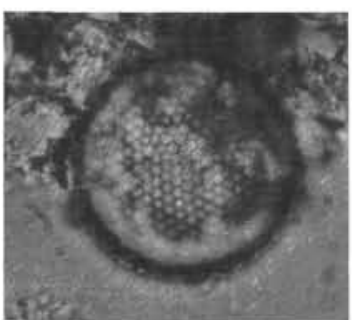

9

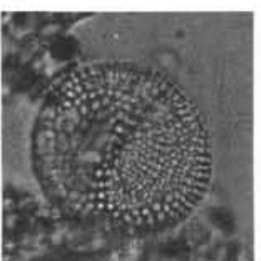

11

12

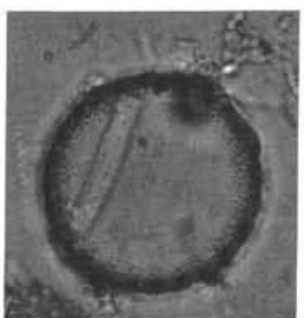

10

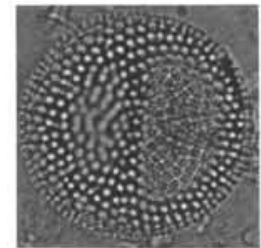

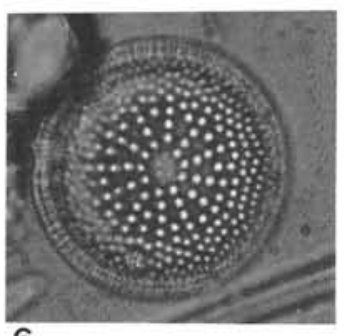

6

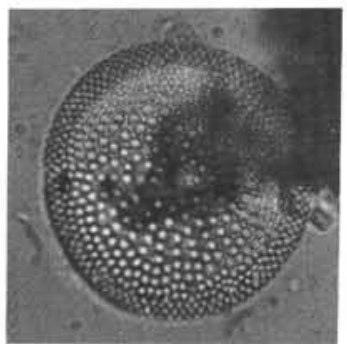

7

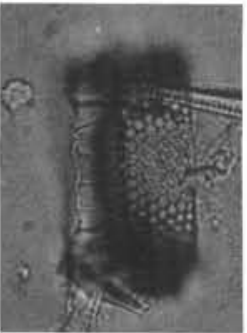

14

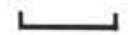

Plate 2. 1, 2. Craspedodiscus coscinodiscus Ehrenberg. 3. Thalassiothrix robusta (Schrader) Akiba. 4. Coscinodiscus vetustissimus Pantocsek $(=$ Azpeitia) $)$ 5. Annellus californicus Tempere (girdle view). 6, 7. Actinocyclus ingens Rattray. 8. Actinocyclus ellipticus Grunow var. elongata Kolbe. 9. Thalassiosira praeconvexa Burckle. 10. Skeletonema spec. (new species, not formally described here). 11, 12. Thalassiosira aff. grunowii Akiba and Yanagisawa. 13. Actinocyclus ehrenbergii Ralfs. 14. Skeletonema species (new species, girdle view).

Scale at bottom right corner of Plate 2 applies to all illustrations, the smallest distance on that scale is 10 microns. 